\title{
Serial Electron Diffraction for Proteins and Small Molecules
}

\author{
R Bücker ${ }^{1}$, P Hogan-Lamarre ${ }^{2}$, P Mehrabi $^{3}$, E Schulz $^{1}$, G Kassier ${ }^{1}$, R Miller $^{4}$ \\ ${ }^{1}$ Max Planck Institute for the Structure and Dynamics of Matter, Hamburg, Germany, ${ }^{2}$ University of \\ Toronto, Toronto, ${ }^{3}$ Max Planck Institute for the Structure and Dynamics of Matter, Hamburg, \\ ${ }^{4}$ University of Toronto, Toronto, Ontario \\ robert.buecker@mpsd.mpg.de
}

Serial crystallography, where diffraction snapshots of a large ensemble of randomly oriented crystals are taken, evades the cumulative damage inherent to rotation diffraction techniques. This approach has facilitated the use of sub-micron crystals in latest-generation X-ray sources, making large classes of small, radiation-sensitive systems such as recalcitrant protein nano-crystals or nano-porous materials amenable to crystallographic structure solution. We recently demonstrated a new scheme for dose-fractionated serial electron nano-crystallography in a scanning TEM, which combines the benefits of serial crystallography with the favorable scattering properties of electrons. It can be conducted in standard microscopes in a highly automated manner and without requiring specific sample delivery devices [1]. I will present our data collection and processing pipeline, show results from protein and smallmolecule crystals, and discuss specific advantages and challenges of a serial crystallography approach as compared to conventional rotation techniques for different types of samples. [1] R. Bücker, P. Hogan-Lamarre, P. Mehrabi, E. C. Schulz, L. A. Bultema, Y. Gevorkov, W. Brehm, O. Yefanov, D. Oberthür, G. H. Kassier, and R. J. D. Miller, Nat. Commun. 11, 996 (2020).

Acta Cryst. (2020). A76, a105 